\title{
Knowledge Transfer Partnerships at the University of the West of England
}

\begin{abstract}
This paper outlines the range of Knowledge Transfer Partnerships (KTPs) undertaken by The University of the West of England and discusses two typical cases in detail. It presents the planned and unplanned benefits that have been identified and realized.

In addition to the organizational improvements we identify the value of KTPs to those employed by the programme in the form of formal qualifications and transferable skills, and the benefits to the university in terms of research and other opportunities.

We also identify the challenges that universities face in attracting and supporting future KTPs.
\end{abstract}

\section{Introduction}

Knowledge Transfer Partnerships (KTPs) offer an attractive way for universities and enterprise to collaborate. Typically, university graduates are employed as KTP Associates to undertake a substantial piece of work for an organization under the guidance of a Workplace Supervisor and a Lead Academic. Not only does the KTP provide the organization with a dedicated resource to complete a significant project but also provides access to a university's store of knowledge and experience. The university gains the opportunity to inject its expertise into the commercial environment directly and to engage in further research. Additionally, the Associate gains valuable vocational experience and is supported through a programme of further study resulting in nationally recognized qualifications including the opportunity to study for a higher degree.

Being part funded and not requiring the diversion of existing human resources away from current organizational activities KTPs have significant advantages over other more traditional consultancy arrangements, particularly for small to medium sized enterprises (SMEs).

Although the benefits of KTPs can be very attractive, it must be stressed that to exploit the opportunities that they can provide to all stakeholders, there needs to be a realization that they must be pro-actively sourced and managed. It is therefore essential to consider the potential barriers to collaboration, many of which are internal to universities. Some of those initial barriers which need to be considered include staffing resources, marketing and business development capabilities and the important aspect of identifying and matching skills and knowledge requirements. These factors are important so that universities are seen to have a 
credible and professional approach to supporting business organizations in achieving their goals and objectives.

This paper outlines the current KTP activities of the Bristol Business School at the University of the West of England (UWE), showing the wide variety and scale of projects that are undertaken. It discusses the goals and achievements of two current KTPs in detail and highlights the unexpected and intangible benefits that have resulted. Finally it presents the value of KTP activity to universities and their staff, in particular, as research opportunities and as rich sources of teaching materials.

\section{The University of the West of England}

UWE has a history of successful KTPs with local and international businesses (http://rbi.uwe.ac.uk/intranet/business/ktp.asp). The department of Strategy and Operations currently supports three out of the five KTPs that are being undertaken within Bristol Business School at the university. Table 1 indicates the types of businesses where these KTPs are in progress and the nature of the project work.

The projects range in value from $£ 100,000$ to just over $£ 200,000$ each with projected savings and benefits of up to $£ 2.6$ million over 5 years. Between $50 \%$ and $67 \%$ of the funding is provided predominantly by the Technology Strategy Board (TSB) with some additional support from the Economic and Social Research Council (ESRC), the remainder being provided by the host organization.

\begin{tabular}{|c|c|}
\hline Business Type & KTP Activity \\
\hline Rural, Non-Profit \\
$\begin{array}{c}\text { Retail, Equipment Service } \\
\& \text { Maintenance }\end{array}$ & ISO9000 / EMAS Certification \\
$\begin{array}{c}\text { Sports Equipment, Design } \\
\& \text { Manufacture }\end{array}$ & Supply Chain Development \\
Marketing Services & \\
Food Distribution & Marketing Technologies Development \\
\hline
\end{tabular}

Table 1, KTP Businesses and Projects 


\section{Rural, Non-Profit (RNP)}

RNP has engaged in a series of KTPs and this is perhaps the best evidence of their real-world benefits. Originally targeted with improving its marketing strategy and activities the later KTPs have grown to encompass business-wide changes to their quality and operational systems and practices.

In 2005 the organization began working toward achieving ISO9000 certification; a considerable undertaking for a non-profit enterprise that operates in a sector where international or industry standards of excellence are not commonplace. Preparation for certification required thorough investigation of the existing business processes. The relatively simplistic method of Business Process Mapping (Hines \& Rich, 1997) was chosen for this purpose; suggested by the Lead Academic for the project whose knowledge and experience of continuous improvement, ISO9000 and business consultancy were well aligned to the KTP's aims.

While mapping the business processes numerous opportunities for immediate improvement were identified, ranging from updating accounting software to simplification of manual processes and the elimination of redundant activities. This approach also enabled the implementation of previously unrealized opportunity such as the initiation of online ordering: this was particularly welcomed by the Chief Executive who commented "it's wonderful, we did $£ 25 \mathrm{k}$ of business last night while we were all asleep!"

KTPs also offer a surprising degree of flexibility which is essential for those businesses that operate in shifting and unpredictable environments. While working toward ISO9000 certification its ultimate value to the organization was brought into question (White et al, 2009) and the Eco-Management and Audit Scheme (EMAS) was identified as a recognized achievement that would probably have greater direct benefit to a rural non-profit organization. Consequently the project was amended and extended to work toward gaining EMAS certification.

Although the project's ultimate aim had changed the fundamental tasks of understanding and improving the core business processes and practices remained. The Associate has acquired widely transferable skills by enrolling on the university's modules in project management and communication training. This has aided the new project's rapid progress and the organization has recently successfully navigated the first formal audit of the new procedures and practices.

The cost and value of ISO9000 to small businesses has received much attention by academia and our work has added to the understanding that it remains a significant challenge to SMEs, particularly non-profits (White et al, 2009). Similarly, there is little theoretical analysis of the implementation of EMAS and the current KTP is providing valuable insights into that process.

In addition to the measurable benefits are numerous intangibles. A formal method of managing projects has been brought into the organization via the Associate. Also, and much to the amusement of the Associate, was the Lead 
Academic's delight upon hearing that he had replaced the battery in the company clock: the clock had been out of action for many years. This is a seemingly innocuous change but one that had been immediately recognized and appreciated by the company staff and, in the Lead Academic's experience, is one that is an effective way of demonstrating commitment to organizational change and improvement.

\section{Food Distribution (FD)}

The KTP project with FD started in October 2008. FD stores and distributes a range of frozen, chilled and ambient food, including fresh meat products, in the South West. Its customer base includes pubs, clubs, hotels, hospitals, nursing homes, restaurants, supermarkets and others. Whilst FD has a loyal customer base and a regional reputation for good service the company is finding increasingly that business is won or lost on price, particularly with public sector clients. In addition, competition is being experienced from national distribution companies who operate on a much larger scale. FD believes that it is strategically important to differentiate the range it offers both from national distributors and other regional competitors.

While more and more consumers start to consider the local factor in their purchasing decision, positive claims about the environmental and social benefits of 'local food' systems are increasingly common (Morgan et al. 2006; EdwardsJones et al. 2008). Supporters claim that 'local food' serves to reduce food miles and greenhouse gas emissions, improve food safety and quality, and provide a chance for supporting the local economy. The company believes that there is an opportunity to differentiate the range it offers from its national competitors by offering 'locally sourced' food to both existing and new customers in the region. The KTP partnership will enable FD to effect a culture change towards a more locally-focused business, whilst maintaining its traditional values of service.

One key part of the project will be gaining a full academically informed understanding of regional customer requirements, demand and pricing for locally sourced food, and supplier requirements and capabilities. However, as EdwardsJones (2008) indicated, how far food has travelled (food miles) is a poor indicator of the environmental and ethical impacts of food production. The benefits of local food can only be assessed through combining spatially explicit life cycle assessment with analysis of social issues. It is essential for FD that the competitive advantage through the promotion of locally sourced products should be sustainable and that their current processes can support the operational implications if locally sourced products are introduced.

The first stage of the project is to look at FD's existing supply chain and develop new distribution and logistics processes to accommodate a greater proportion of chilled (rather than frozen food) and substantially increased numbers 
of smaller customers and suppliers. Value stream mapping tools were chosen to map the end to end supply chain. Through the supply chain mapping, the Associate identified that for some products such as frozen chips, no value is being added for the vast majority of time that products are within the defined supply. In addition, the internal lead times are, on average, long, with supplier lead times considerably shorter. It also showed that there is a very high level of available stock which results in low stock turnover rate, and there is a fundamental dislocation between actual demand and actual purchases required. Although many factors contribute to the high stock level, such as suppliers' promotion and long product shelf life, further analysis indicates that for a single product line, FD could save up-to $£ 17,000$ if customer demand, ordering cost, stock holding cost, and price discount were incorporated into the ordering quantity decision making process.

Through the value stream mapping and analysis of different operations process using role activity diagrams, the Associate identified that in the butchery section, the processes used to fulfill a meat order are labour intensive and that a significant amount of time is spent on each order that did not add value. The analysis also highlighted that the current process is prone to error at a number of points due to the reliance on butchers to complete an order from start to finish. The Associate also found that the main causes for the discontinuation of some newly launched chilled products are low shelf life, high minimum order quantity and low demand. These criteria could be considered in the decision-making of launching new chilled products in future.

Overall, the analysis highlights that there is a requirement within the business to pay closer attention to its operations. Although the KTP project is still in its early stage, a number of recommendations have been made based on these findings. The majority of these recommendations can be quickly implemented and are expected to bring significant benefits to FD.

\section{Summary}

KTPs are capable of delivering significant bottom-line benefits to organizations and may be more attractive than other consultancy arrangements, particularly for SMEs. Not only are they capable of delivering substantial benefits, such as achieving EMAS certification or re-engineering supply chains, but also of identifying and implementing other unexpected tangible and intangible benefits.

The cases outlined above are significant projects, greater in scale than perhaps many SMEs would consider embarking upon. Shorter KTPs for projects of between 10 and 40 weeks are now available, providing greater flexibility and attractiveness to smaller enterprises.

For universities and their staff, KTPs offer stimulating working environments, valuable research opportunities and widening networks and contacts. KTPs may 
be a source of interesting cases for students to study, particularly since they are likely to involve organizations with which the student is familiar.

KTP income is a contributor to the Research Assessment Exercise (RAE), one may also consider how knowledge transfer between university and industry may be further recognized and developed for future Research Excellence Framework (REF) assessments. Ultimately KTPs and other knowledge transfer mechanisms are drivers for collaboration that is seen as a necessary dimension of both regional and national economic and intellectual success for the future (Abd Razak and Saad, 2007; Cooke, 2002; Morgan, 1997).

The growing importance of university-commerce knowledge transfer will require universities to maximize their presence within local and regional business environments. It will become increasingly important to be able to provide the wide range of appropriately skilled and experienced academic resources that organizations require. Possession of such an eclectic resource may be the competitive advantage that enables a university's collaborative efforts to flourish.

\section{References}

Abd Razak, A., \& Saad, M. (2007). The role of universities in the evolution of the Triple Helix culture of innovation network: The case of Malaysia. International. Journal of Technology Management and Sustainable Development, 6(3), 211-225.

Cooke, P. (2002). Knowledge Economies: Clusters, learning and cooperative advantage. London and New York: Routledge.

Edwards-Jones, G., Canalsb, L.M., Hounsomec, N., Truningerd, M., Koerbera, G., Hounsomee, B., Crossa, P., Yorka, E.H., Hospidob, A., Plassmanna, K., Harrisa, I.M., Edwardse, R.T., Dayd, G.A.S., Tomosc, A.D., Cowellb, S.J. and Jonesa, D.L., (2008). Testing the assertion that 'local food is best': the challenges of an evidence-based approach. Trends in Food Science \& Technology, 19, 265-274

Hines, P., Rich, N. (1997) The Seven Value Stream Mapping Tools. International Journal of Operations and Production Management, 17(1), 46-64.

Morgan, K. (1997). 'The Learning Region: Institutions, Innovation and Regional Renewal'. Regional Studies, 31, 491-503.

Morgan, K., Marsden, T., \& Murdoch, J. (2006). Worlds of food. Place, power and provenance in the food chain. Oxford: Oxford University Press.

White, G.R.T., Samson, P., Rowland-Jones, R., Thomas, A.J. (2009). The Implementation of a Quality Management System in the Not-for-Profit Sector. The TQM Magazine, 20(3), 273283 . 